\title{
Transient dynamics in electric power system with dc transmission: Fractal growth in stability boundary
}

\author{
$\operatorname{AUTHOR}(\mathrm{S}):$ \\ Susuki, Yoshihiko; Hikihara, Takashi
}

\section{CITATION:}

Susuki, Yoshihiko ... [et al]. Transient dynamics in electric power system with dc transmission: Fractal growth in stability boundary. IEE Proceedings - Circuits, Devices and Systems 2005, 152(2): 159-164

\section{ISSUE DATE:}

2005

URL:

http://hdl.handle.net/2433/49152

\section{RIGHT:}

This paper is a postprint of a paper submitted to and accepted for publication in [IEE Proceedings - Circuits, Devices and Systems] and is subject to Institution of Engineering and Technology Copyright. The copy of record is available at IET Digital Library; この論文は出版社版でありません。引用の際には出版社版をご確認ご利用ください。; This is not the published version. Please cite only the published version. 
Transient dynamics in electric power system with dc transmission: Fractal growth in stability boundary

\author{
Yoshihiko Susuki and Takashi Hikihara
}

Department of Electrical Engineering, Kyoto University

Submission of Revised Version to IEE Proceedings - Circuits, Devices and Systems

Reference Paper Number 42626

\title{
Corresponding Author
}

Yoshihiko Susuki

Hikihara Laboratory, Department of Electrical Engineering, Kyoto University Katsura, Nishikyo-ku, Kyoto, 615-8510, JAPAN

Phone: +81-75-383-2243

Fax: $+81-75-383-2238$

E-mail: susuki@dove.kuee.kyoto-u.ac.jp 


\begin{abstract}
This paper is concerned with transient dynamics and stability of an electric power system with dc transmission. Nowadays dc transmission systems are widely applied to conventional electric power systems. However, transient dynamics of ac/dc power systems, affected by active power which flows into dc transmissions, is not entirely understood. In this paper an unsymmetrical swing equation system is derived to analyze the transient dynamics of an ac/dc power system. The unsymmetry implies an unidirectional component of an external forcing which corresponds to both dc power and exciting power swing. This paper discusses a stability boundary and its qualitative change in the unsymmetrical swing equation system. The understanding of the stability boundary plays an important clue to grasp the transient dynamics of the ac/dc power system. We now reveal fractal structure growth in the stability boundary caused by the change of dc power flow. The existence of fractal boundary implies that the system behaviour becomes chaotic and unpredictable depending on the operation of the dc transmission.
\end{abstract}




\section{Introduction}

Nowadays dc transmission systems are widely applied to conventional electric power systems $[1,2,3,4,5]$. The dc transmissions have much stronger advantages: they can connect different ac power systems under desynchronized operation and achieve rapid active power flow control. In particular power swing damping control is discussed based on dc power modulation $[4,5,6]$. However, transient dynamic of ac/dc power systems, affected by active power flow into dc transmissions and exciting power swing, is not entirely understood. The understanding of the dynamics is much inevitable for operation and control of future power supply networks including various dc-based apparatuses.

The present paper discusses transient dynamics, affected by the dc power flow, of an ac/dc power system based on an unsymmetrical swing equation system. The unsymmetry implies an unidirectional component of an external forcing which corresponds to both the dc power and exciting power swing. The swing equation system has the common mathematical structure to various physical and engineering systems: forced pendulums [7], Josephson junction circuits [8] and phase-locked loops $[9,10]$. The dynamical behaviour affected by the dc external force, however, has been unsolved for these systems although some studies on Duffing equation with unsymmetrical force were reported in $[11,12]$. The dynamical behaviour now remains in an open problem of not only power systems dynamics and also nonlinear oscillation theory.

As an important aspect of dynamical behaviour we consider a stability boundary and its qualitative change in the swing equation system. The stability boundaries imply basin boundaries of stable equilibrium points and periodic solutions which correspond to post-fault steady states of the ac/dc power system. These concept is widely recognized as a mathematical foundation of transient stability analysis of electric power systems [13]. We show in this paper that fractal structure grows in the stability boundary of the 
swing equation system caused by the dc external forcing. The fractal growth indicates in the contexts of power systems engineering that we cannot inherently predict the transient behaviour of the ac/dc system if its post-fault state is placed around the boundary. The fractal structure is numerically and analytically discussed based on geometric theory of dynamical systems $[14,15,16]$. The preliminary discussion in the paper has been reported in [17].

The organization of this paper is as follows. Sec. 2 introduces an unsymmetrical swing equation system to analyze transient dynamics of an electric power system with dc transmission. This section also provides us with some dynamical properties of the system. Sec. 3 shows a bifurcation diagram of the swing equation system. The diagram implies some information about steady states and qualitative changes of phase portraits in the system: phase and basin portraits show global structures of solution space which completely characterize stability boundaries. In Sec. 4 we discuss a stability boundary in which fractal structure grows by a global homoclinic bifurcation. The discussion is numerically and analytically performed based on several phase and basin portraits. Sec. 5 concludes this paper with summary of our present results.

\section{System model and mathematical formulation}

\subsection{Introduction to swing equation system}

Figure 1 shows a system model of an electric power system with dc transmission. The ac/dc system is introduced based on the practical system $[3,4,5]$. In Fig. 1 the dc transmission is closely connected to the bus of generator. A sinusoidal power swing, which exists in the ac transmission, is assumed to flow into the bus. The power swing is often observed in the practical systems; It is therefore expected to be damped by the dc power modulation $[4,5,6]$. In Fig. 1 the dc transmission is assumed to be ideally operated. The main reason is that this paper aims to clarify the dynamics of 
the generator affected by the active power flow into the dc transmission. It does not loose the generality of discussion.

A swing equation system is derived to analyze transient dynamics of the ac/dc power system in Fig. 1. In the figure $p_{\mathrm{e}(\mathrm{ac})}$ denotes the active power which flows into the infinite bus, and can be approximately given by

$$
p_{\mathrm{e}(\mathrm{ac})}=b \sin \delta-\varepsilon b \cos \Omega \tau
$$

where the physical meanings of variables and parameters are shown by Tab. 1 . On the other hand, $p_{\mathrm{e}(\mathrm{dc})}$ denotes the active dc power flow. The dynamics of the generator is then represented by the following system of ordinary differential equation:

$$
\left\{\begin{array}{l}
\frac{\mathrm{d} \delta}{\mathrm{d} \tau}=\omega \\
\frac{\mathrm{d} \omega}{\mathrm{d} \tau}=-D \omega+p_{\mathrm{m}}-\left(p_{\mathrm{e}(\mathrm{ac})}+p_{\mathrm{e}(\mathrm{dc})}\right) .
\end{array}\right.
$$

In the system the external force $\left(p_{\mathrm{m}}-p_{\mathrm{e}(\mathrm{dc})}\right)+\varepsilon b \cos \Omega \tau$ is unidirectional; It is called an unsymmetrical forcing. The derivation of the system (2) is the almost same as that of conventional swing equation systems seen in $[2,18]$.

The swing equation system (2) is relevant to analyzing transient dynamics of the ac/dc power system. Transient dynamics and stability of ac power systems are essentially governed by active power-balance among generators and dc transmissions. From this phenomenological point of view, transient stability in multi-machine ac/dc systems is discussed via similar swing equation system including control setup of the rectifiers [19]. Additionally swing dynamics in a single generator-infinite bus system with UPFC is considered based on the same mathematical model as the system (2) [18]. The transient dynamics of the ac/dc system can be thus discussed based on the swing equation system (2). 


\subsection{Dynamical properties of swing equation system}

This section provides us with some dynamical properties of the swing equation system (2). The goal of this study is to clarify how the dc power flow affects the transient dynamics of the ac/dc power system; Our aim in the paper is to illustrate fractal growth in the stability boundary of the system (2). To do that we here arrange several fundamental characteristics of the system (2) in the contexts of geometric theory of dynamical systems: readers can refer to introductory texts $[14,15,16]$.

First the system (2) has two kinds of periodic solutions $(\delta(\tau), \omega(\tau))$ because of the periodicity of $2 \pi$ for $\delta(\tau):(\delta, \omega) \in \mathbb{S}^{1} \times \mathbb{R}\left(\mathbb{S}^{1}=\{\delta \mid-\pi \leq \delta<\pi(\bmod 2 \pi)\}\right)$. In $[20]$ one is a first kind periodic solution which possesses following property:

$$
\delta\left(\tau+\frac{2 \pi}{\Omega}\right) \equiv \delta(\tau), \quad \omega\left(\tau+\frac{2 \pi}{\Omega}\right) \equiv \omega(\tau), \quad \text { for } \tau \in \mathbb{R}
$$

and another is a second kind periodic solution satisfying

$$
\delta\left(\tau+\frac{2 \pi}{\Omega}\right) \equiv \delta(\tau)+2 m \pi, \quad \omega\left(\tau+\frac{2 \pi}{\Omega}\right) \equiv \omega(\tau), \quad \text { for } \tau \in \mathbb{R} \text { and } m \in \mathbb{Z}
$$

where each solution keeps the period $2 \pi / \Omega$ of exciting power swing constant.

Second we introduce a discrete dynamical system on the cylindrical phase plane via stroboscopic sampling of solution in the system (2) at $\tau=2 n \pi / \Omega(n \in \mathbb{Z})$. Through the stroboscopic sampling any solution of the system (2) defines a discrete dynamical system on the cylindrical phase plane $(\delta, \omega) \in \mathbb{S}^{1} \times \mathbb{R}$, or a map on $\mathbb{S}^{1} \times \mathbb{R}$ into itself:

$$
\mathcal{F}_{\lambda} \quad: \quad \mathbb{S}^{1} \times \mathbb{R} \rightarrow \mathbb{S}^{1} \times \mathbb{R}
$$

where $\lambda$ denotes the set of parameters in the system (2). By applying the discrete system $\mathcal{F}_{\lambda}$ we can examine global structures of solution space, in particular, stability boundaries of the system (2) on the cylindrical phase plane. The discrete system then transforms above periodic solutions to fixed points at which associated linearized maps decide the kind of fixed points' stability. 
Lastly an existence condition of doubly asymptotic points is given for invariant manifolds in the system (2). The system (2) is one of one-degree-of-freedom periodically perturbed Hamiltonian systems. Melnikov's methods therefore has a potential to clarify some dynamical characteristics of the system $(2)[8,9,14,15]$. The Hamiltonian system, which $D=0, p_{\mathrm{m}}-p_{\mathrm{e}(\mathrm{dc})}=0$ and $\varepsilon=0$, has the following homoclinic orbit:

$$
\left(\delta_{0}^{ \pm}(\tau), \omega_{0}^{ \pm}(\tau)\right)=( \pm 2 \arcsin (\tanh (\sqrt{b} \tau)), \pm 2 \sqrt{b} \operatorname{sech}(\sqrt{b} \tau))
$$

Under sufficiently small perturbation $D, p_{\mathrm{m}}-p_{\mathrm{e}(\mathrm{dc})}$ and $\varepsilon$, we now obtain the existence condition of doubly asymptotic points in the region $\omega>0$ as follows:

$$
\varepsilon b \geq\left|\frac{4 D \sqrt{b}}{\pi}-\left(p_{\mathrm{m}}-p_{\mathrm{e}(\mathrm{dc})}\right)\right| \cdot \cosh \left(\frac{\Omega \pi}{2 \sqrt{b}}\right) .
$$

While, the existence of doubly asymptotic points in the region $\omega<0$ is also conditioned as follows:

$$
\varepsilon b \geq\left|\frac{4 D \sqrt{b}}{\pi}+\left(p_{\mathrm{m}}-p_{\mathrm{e}(\mathrm{dc})}\right)\right| \cdot \cosh \left(\frac{\Omega \pi}{2 \sqrt{b}}\right) .
$$

We should note that the obtained conditions correspond to the existence of heteroclinic points if the system (2) is defined on the phase plane [21]. The derivation of the conditions (7) and (8) is confirmed in [9].

\section{Bifurcation diagram}

Figure 2 shows the bifurcation diagram in the control plane $\left(p_{\mathrm{m}}-p_{\mathrm{e}(\mathrm{dc})}, \varepsilon\right)$ of the discrete dynamical system (5) at the following parameters:

$$
D=0.05, \quad b=0.7, \quad \Omega=0.5
$$

where $\Omega$ corresponds to $0.86 \mathrm{~s}$ in the practical time. These values are fixed based on the practical system $[3,4,5]$. In Fig. 2 the curves Melnikov criteria present the boundary in which doubly asymptotic points appear; Those properties are identical to those of 
heteroclinic points on the phase plane. The criteria are induced from the conditions (7) and (8). The curves Fold (Sync.) are the boundaries correspond to the occurrence of synchronization into second kind limit cycles which the system (2) under $\varepsilon=0$ possesses depending on $p_{\mathrm{m}}-p_{\mathrm{e}(\mathrm{dc})}$ : from technical point of view its synchronized state represents an unacceptable state of the ac/dc power system shown in Fig. 3(c). In addition the curves Period-Doubling (Sync.) correspond to the threshold sets of the period-doubling bifurcation of second kind completely stable fixed points. The bifurcation sets Fold B show the generation of first kind resonant solutions through fold bifurcation. The resonant solutions usually have larger amplitude of oscillation than that of the nonresonant solutions as shown in Fig. 3. Furthermore the curves Period-Doubling C exhibit the period-doubling bifurcation sets with respect to completely stable resonant solutions. Fig. 3(d) also shows a second kind invariant closed curve in the discrete dynamical system (5); It coincides with another undesirable condition of the ac/dc power system.

\section{Global bifurcation and fractal growth in stability bound-}

\section{$\operatorname{ary}$}

This section discusses a global bifurcation scenario caused by the change of dc external force $p_{\mathrm{m}}-p_{\mathrm{e}(\mathrm{dc})}$ based on several phase and basin portraits. We show in this section that the global bifurcation generates true fractal structure in the stability boundary.

\subsection{Global bifurcation scenario by dc external force $p_{\mathrm{m}}-p_{\mathrm{e}(\mathrm{dc})}$}

We here investigate the global bifurcation scenario according to the change of $p_{\mathrm{m}}-p_{\mathrm{e}(\mathrm{dc})}$. Fig. 4 shows the phase and basin portraits of the discrete dynamical system (5) under $\left(p_{\mathrm{m}}-p_{\mathrm{e}(\mathrm{dc})}, \varepsilon\right)=(0.06,0.1)$ and $(0.07,0.1)$. In each phase portrait the symbol ${ }^{k} \mathrm{D}_{(i)}^{j}$ $\left({ }^{j} \mathrm{~S}_{(i)}^{k}\right.$, resp.) denotes the $i$-th directly unstable (completely stable, resp.) $j$-th kind 
$k$-periodic point. The solid line in the figures represents the unstable manifold of the resonant fixed point ${ }^{1} \mathrm{D}_{(2)}^{1}$ and the broken line its stable manifold. While in each basin portrait the region is coloured white for the non-resonant point ${ }^{1} S_{(1)}^{1}$ and black for the resonant one ${ }^{1} S_{(2)}^{1}$. In addition the gray region shows the basin of the second kind stable point ${ }^{2} \mathrm{~S}_{(2)}^{1}$ under $p_{\mathrm{m}}-p_{\mathrm{e}(\mathrm{dc})}=0.06$ and the invariant closed curve, denoted by ICC in Fig. $4(\mathrm{~b})$, under $p_{\mathrm{m}}-p_{\mathrm{e}(\mathrm{dc})}=0.07$. Fig. 4 here describes the qualitative change of phase portraits by the global bifurcation scenario. Fig. 5 shows the global bifurcation scenario by the increase of $p_{\mathrm{m}}-p_{\mathrm{e}(\mathrm{dc})}$. In Fig. $5(\mathrm{a})$ the unstable manifold of ${ }^{1} \mathrm{D}_{(2)}^{1}$ converges to ${ }^{1} \mathrm{~S}_{(2)}^{1}$, and it does not intersect the stable manifold of ${ }^{1} \mathrm{D}_{(2)}^{1}$. The small increase of $p_{\mathrm{m}}-p_{\mathrm{e}(\mathrm{dc})}$ raises the intersection of the stable and unstable manifolds of ${ }^{1} \mathrm{D}_{(2)}^{1}$ shown in Fig. 5(b). These points are called doubly asymptotic points; The qualitative change of the phase portraits is well-known as a global homoclinic bifurcation.

\subsection{Self-similar basin boundary by the global bifurcation}

The basin boundary of ${ }^{1} \mathrm{~S}_{(2)}^{1}$ shows the self-similar structure caused by the global bifurcation. Fig. 6 shows the magnifications of basin portraits in the neighborhood of ${ }^{1} D_{(2)}^{1}$. In Figs. 5(a) and 6(a) the basin boundary of ${ }^{1} \mathrm{~S}_{(2)}^{1}$ consists of the stable manifold of ${ }^{1} \mathrm{D}_{(2)}^{1}$, and the basin boundary is smooth. When $p_{\mathrm{m}}-p_{\mathrm{e}(\mathrm{dc})}$ increases, in Fig. $6(\mathrm{~b})$ the basin boundary appears to be complicated. Fig. 6(b) also shows that the boundary has the self-similarity for the magnification of the small rectangles.

\subsection{Doubly asymptotic points and fractal basin boundary}

The self-similar basin boundary does not necessary become fractal on the cylindrical phase plane. Generally speaking complicated boundaries possibly exist when doubly asymptotic points exist in the phase plane. In particular if such boundaries exist in the phase plane and transversal homoclinic points appear, the boundaries become fractal [22]. The criterion cannot be, however, applied to the system (2) because the system 
is defined on the cylindrical phase plane [21]. We thus need to reconsider the basin boundary structure based on geometric theory of differential equations.

The basin boundary in Fig. 6(b) has, however, the infinite self-similar structure which is qualitatively identical to fractal structure caused by transversal homoclinic points on the phase plane. In the figure all doubly asymptotic points are based on the invariant manifolds of the first kind fixed point ${ }^{1} \mathrm{D}_{(2)}^{1}$. Fig. $5(\mathrm{~b})$ also shows that the points consist of the intersection between the invariant manifolds which are described only in the rectangle region of this figure. These properties imply that the doubly asymptotic points qualitatively coincide with the transversal homoclinic points on the phase plane. We hence conclude that the self-similar boundary of ${ }^{1} S_{(2)}^{1}$ becomes fractal.

\subsection{Physical interpretation of fractal basin boundary - From the view- point of power systems dynamics}

The fractal stability boundary indicates that the system behaviour becomes chaotic and unpredictable depending on the operation of the dc transmission. In Fig. 4 the stable manifold of ${ }^{1} \mathrm{D}_{(2)}^{1}$ becomes a part of the basin boundary of the non-resonant point ${ }^{1} S_{(1)}^{1}$, that is, the stability boundary of the ac/dc power system. In addition ${ }^{1} S_{(2)}^{1}$ corresponds to an undesirable operation because of its large amplitude of oscillation as shown in Fig. 3. The fractal structure thus grows in the stability boundary of the ac/dc power system. This implies that we cannot precisely predict the transient behaviour of the ac/dc system when its state is placed around the boundary by some accidental faults.

We should note that the fractal boundary is a native dynamical feature of the ad/dc power system under external power disturbance. The system (2) is derived under the assumption that the dc transmission is ideally operated. The mechanism of the fractal growth therefore originates from not switching operation and related instability in ac-dc 
converters but native active power-balance relation among the main generator, infinite bus and dc transmission. As mentioned in Sec. 2 the power-balance relation essentially governs transient dynamics of the ac/dc power system. We can hence state that its transient behaviour inherently becomes indeterminate although the dc apparatuses are safely operated. The authors believe that the obtained feature will be recognized in future power supply networks involving various dc-based apparatuses.

\section{Conclusions}

An aspect of the transient dynamics was numerically and analytically discussed in an electric power system with dc transmission. The swing equation system (2) has the fractal basin boundary depending on the dc external forcing. In $[23,24,25]$ the existence of fractal basin boundaries has been already reported for the similar swing equation systems. The fractal basin boundaries were, however, considered based on numerical simulation or Melnikov's method. As discussed in Sec. 2 the Melnikov's method reveal only the existence of doubly asymptotic points which are identical to heteroclinic points in the phase plane, and do not therefore justify the existence of the fractal basin boundaries. On the other hand, in this paper, we first characterize the fractal basin boundary of the swing equation system (2) based on doubly asymptotic structure in the cylindrical phase plane. Furthermore, in [26, 27], complicated stability boundaries were reported for multi-degree-of-freedom swing equation systems derived to analyze transient stability of small prototype ac systems. It was then concluded that the boundaries will not become fractal, which are called truncated-fractal [26]. The fractal boundary in the paper therefore shows a new indeterminate factor in power systems dynamics and stability.

Our further direction is to establish an analytical method for evaluating stability boundaries in non-autonomous systems with resonant solutions. In [28] we proposed an 
analytical criterion for stability boundaries in non-autonomous systems based on Melnikov's method. Unfortunately, the criterion is not effective for the stability boundaries in the swing equation system (2) which possesses some resonant solutions and fractal basin boundaries: their typical examples were discussed in Sec. 4. The other paper will propose an analytical method for evaluating the stability boundaries with respect to the resonant solutions based on subharmonic Melnikov functions [29].

\section{Acknowledgments}

The authors greatly appreciate his fruitful discussions with Professor Yoshisuke Ueda. They are also grateful to Professor Tsuyoshi Funaki for valuable discussions about power systems dynamics. This research was supported in part by the Ministry of Education, Culture, Sports, Sciences and Technology in Japan, under The 21st Century COE Program No. 14213201, and Grant-in-Aid for Scientific Research (C) No. 14550264, 2003.

\section{References}

[1] PADIYAR, K. R.: 'HVdc power transmission systems' (John Wiley \& Sons, New York, 1990)

[2] KUNDUR, P.: 'Power system stability and control' (McGraw-Hill, New York, 1994)

[3] SEKInE, Y., KATO, S., MOTOKI, T. and ITO, S.: 'Kii Channel HVdc Link between Shikoku and Kansai Electric Power Companies by submarine cables', CIGRE Tokyo Symposium, 1995, 220-04

[4] FUJIWARA, T., MATSUNO, K., INOUE, Y. and HAYASHI, T.: 'Study of power modulation and fast margin angle control in Kii Channel HVdc Link', ibid., 1995, $410-05$ 
[5] TOMIYAMA, K., SATO, M., YAMAJI, K., SEKITA, M., and GOTO, M.: 'Power swing damping control by HVdc power modulation in ac/dc hybrid transmission system', T. Institute of Electrical Engineers, Japan, 1997, 117-B (7) pp. 938-944 (in Japanese); Electr. Eng. Jpn., 1998, 124 (3) pp. 10-18

[6] CRESAP, R. L., SCOTt, D. N., MitTelStADT, W. A., and TAYLOR, C. W.: 'Operating experience with modulation of the Pacific HVdc Intertie', IEEE T. Power Ap. Syst., 1978, PAS-97 (4) pp. 1053-1059

[7] D'HUMieres, D., BEASLEy, M. R., HUBERMAN, B. A., and LIBCHARBER, A.: 'Chaotic states and routes in the forced pendulum', Phys. Rev. A, 1982, 26 (6) pp. $3483-3496$

[8] SALAM, F. M. A., and SASTRY, S. S.: 'Dynamics of the forced Josephson junction circuit: The regions of chaos', IEEE T. Circuits Syst., 1985, CAS-32 (8) pp. 784796

[9] ENDO, T., and CHUA, L. O.: 'Chaos from phase-locked loops', IEEE T. Circuits Syst., 1988, 35 (8) pp. 987-1003

[10] OHNO, W., ENDO, T., and UEDA, Y.: 'Extinction and intermittency of the chaotic attractor via crisis in phase-locked loop equation with periodic external forcing term', T. Institute of Electronics, Information and Communication Engineers, 1999, J82-A (5) pp. 627-636 (in Japanese)

[11] HAYASHI, C.: 'Nonlinear oscillations in physical systems' (McGraw-Hill, New York, 1964)

[12] UEDA, Y.: 'Explosion of strange attractors exhibited by Duffing's equation', Nonlinear Dynamics, Annals of the New York Academy of Sciences, 1980, 357 pp. $422-434$ 
[13] CHIANG, H. -D., HIRSCH, M. W., and WU, F. F.: 'Stability regions of nonlinear autonomous dynamical systems', IEEE T. Automat. Contr., 1988, 33 (1) pp. 16-27

[14] GUCKENHEIMER, J., and HOLMES, P.: 'Nonlinear oscillations, dynamical systems, and bifurcations of vector fields' (Springer-Verlag, New York, 1983)

[15] WIGGNIS, S.: 'Introduction to applied nonlinear dynamical systems and chaos' (Springer-Verlag, New York, 1990)

[16] THOMPSON, J. M. T., and STEWART, H. B.: 'Nonlinear dynamics and chaos' (John Wiley \& Sons, London, 2002)

[17] SUSUKI, Y., and HIKIHARA, T.: 'Swing phenomena and global structures of solution space in an electric power system with dc transmission'. 9th Workshop on Nonlinear Dynamics of Electronic Systems (NDES2001), Jun. 2001, Delft, The Netherlands, pp. 133-136

[18] MACHOWSKI, J., BIALEK, J. W., and BUMBY, J. R.: 'Power system dynamics and stability' (John Wiley \& Sons, New York, 1997)

[19] PAI, M. A., PADIYAR, K. R., and RADHAKRISHNA, C.: 'Transient stability analysis of multi-machine ac/dc power systems via energy-function method', IEEE T. Power Ap. Syst., 1981, PAS-100 (12) pp. 5027-5035

[20] UEDA, Y, AKAMATA, Y., and NANAHARA, T.: 'Phase-locked loop with delay time -Computer experiment on pull-in range-', T. Institute of Electronics and Communication Engineers, 1978, 61-B (6) pp. 457-463 (in Japanese)

[21] UEDA, Y.: Personal Communication, 1999

[22] MOON, F. C., and LI, G. -X.: 'Fractal basin boundaries and homoclinic orbits for periodic motion in a two-well potential', Phys. Rev. Lett., 1985, 55 (14) pp. $1439-1442$ 
[23] GWINN, E. G., and WESTERVELT, R. M.: 'Intermittent chaos and lowfrequency noise in the driven damped pendulum', Phys. Rev. Lett., 1985, 54 (15) pp. $1613-1616$

[24] GREBOGI, C., OTT, E., and YORKE, J. A.: 'Basin boundary metamorphoses: Changes in accessible boundary orbits', Physica D, 1987, 24 pp. 243-262

[25] ENDO, T., and CHUA, L. O.: 'Bifurcation diagrams and fractal basin boundaries of phase-locked loop circuits', IEEE T. Circuits Syst., 1990, 37 (4) pp. 534-540

[26] VARGHESE, M., and THORP, J. S.: 'An analysis of truncated fractal growths in the stability boundaries of three-node swing equations', IEEE T. Circuits Syst., 1988, 35 (7) pp. 825-834

[27] HASEGAWA, Y., and UEDA, Y.: 'Global basin structure of attraction of two degrees of freedom swing equation system', Int. J. Bifurcat. Chaos, 1999, 9 (8) pp. $1549-1569$

[28] SUSUKI, Y., and HIKIHARA, T.: 'An analytical criterion for stability boundaries of non-autonomous systems based on Melnikov's method', T. Institute of Systems, Information and Control Engineers, 2002, 15 (11) pp. 586-592

[29] SUSUKI, Y., and HIKIHARA, T.: 'Stability boundaries analysis of nonautonomous systems with resonant solutions based on subharmonic Melnikov function', American Control Conference (ACC2004), Jun. 2004, Boston, United States (to appear) 
Table 1: Physical meanings of variables and parameters

\begin{tabular}{ll}
\hline$\delta$ & rotor position with respect to synchronously reference axis \\
\hline$\omega$ & rotor speed difference relative to system angular frequency \\
\hline$\tau$ & normalized time \\
\hline$D$ & damping coefficient in generator \\
\hline$b$ & critical value of single generator-infinite bus system \\
\hline$p_{\mathrm{m}}$ & mechanical input power to the generator \\
\hline$p_{\mathrm{e}(\mathrm{dc})}$ & active power flow into dc transmission \\
\hline$\varepsilon$ & ratio of exciting power swing to critical value $b$ \\
\hline$\Omega$ & angular frequency of exciting power swing \\
\hline
\end{tabular}




\section{List of captions to illustrations}

Figure 1 System model of electric power system with dc transmission

Figure 2 Bifurcation diagram of discrete dynamical system (5) at $D=0.05, b=0.7$ and $\Omega=0.5$

Figure 3 Waveforms of rotor position at steady states for swing equation system (2). The symbol $\times$ stands for the stroboscopic point through corresponding sampling $\tau=2 \pi / \Omega$.

(a) First kind non-resonant solution at $\left(p_{\mathrm{m}}-p_{\mathrm{e}(\mathrm{dc})}, \varepsilon\right)=(0.06,0.1)$

(b) First kind resonant solution at $\left(p_{\mathrm{m}}-p_{\mathrm{e}(\mathrm{dc})}, \varepsilon\right)=(0.06,0.1)$

(c) Second kind solution at $\left(p_{\mathrm{m}}-p_{\mathrm{e}(\mathrm{dc})}, \varepsilon\right)=(0.06,0.1)$

(d) Second kind invariant closed curve at $\left(p_{\mathrm{m}}-p_{\mathrm{e}(\mathrm{dc})}, \varepsilon\right)=(0.07,0.1)$

Figure 4 Phase (upper) and basin (lower) portraits of discrete dynamical system (5) at $\left(p_{\mathrm{m}}-p_{\mathrm{e}(\mathrm{dc})}, \varepsilon\right)=(0.06,0.1)$ and $(0.07,0.1)$. In the phase portraits the solid line represents the unstable manifold of ${ }^{1} D_{(2)}^{1}$ and the broken line represents its stable manifold. In the basin portraits the region is coloured white for ${ }^{1} \mathrm{~S}_{(1)}^{1}$, black for ${ }^{1} \mathrm{~S}_{(2)}^{1}$, and gray for ${ }^{2} \mathrm{~S}_{(2)}^{1}$ under $p_{\mathrm{m}}-p_{\mathrm{e}(\mathrm{dc})}=0.06$ and ICC under $p_{\mathrm{m}}-p_{\mathrm{e}(\mathrm{dc})}=0.07$.

(a) $\left(p_{\mathrm{m}}-p_{\mathrm{e}(\mathrm{dc})}, \varepsilon\right)=(0.06,0.1)$

(b) $\left(p_{\mathrm{m}}-p_{\mathrm{e}(\mathrm{dc})}, \varepsilon\right)=(0.07,0.1)$

Figure 5 Sequence of phase portraits at $\left(p_{\mathrm{m}}-p_{\mathrm{e}(\mathrm{dc})}, \varepsilon\right)=(0.06,0.1)$ and $(0.07,0.1)$

(a) $\left(p_{\mathrm{m}}-p_{\mathrm{e}(\mathrm{dc})}, \varepsilon\right)=(0.06,0.1)$

(b) $\left(p_{\mathrm{m}}-p_{\mathrm{e}(\mathrm{dc})}, \varepsilon\right)=(0.07,0.1)$

Figure 6 Magnified sequences of basin portraits of neighboring domain of ${ }^{1} D_{(2)}^{1}$. Each magnified basin portraits is obtained inside the small square in this figure. 
(a) $\left(p_{\mathrm{m}}-p_{\mathrm{e}(\mathrm{dc})}, \varepsilon\right)=(0.06,0.1)$

(b) $\left(p_{\mathrm{m}}-p_{\mathrm{e}(\mathrm{dc})}, \varepsilon\right)=(0.07,0.1)$ 


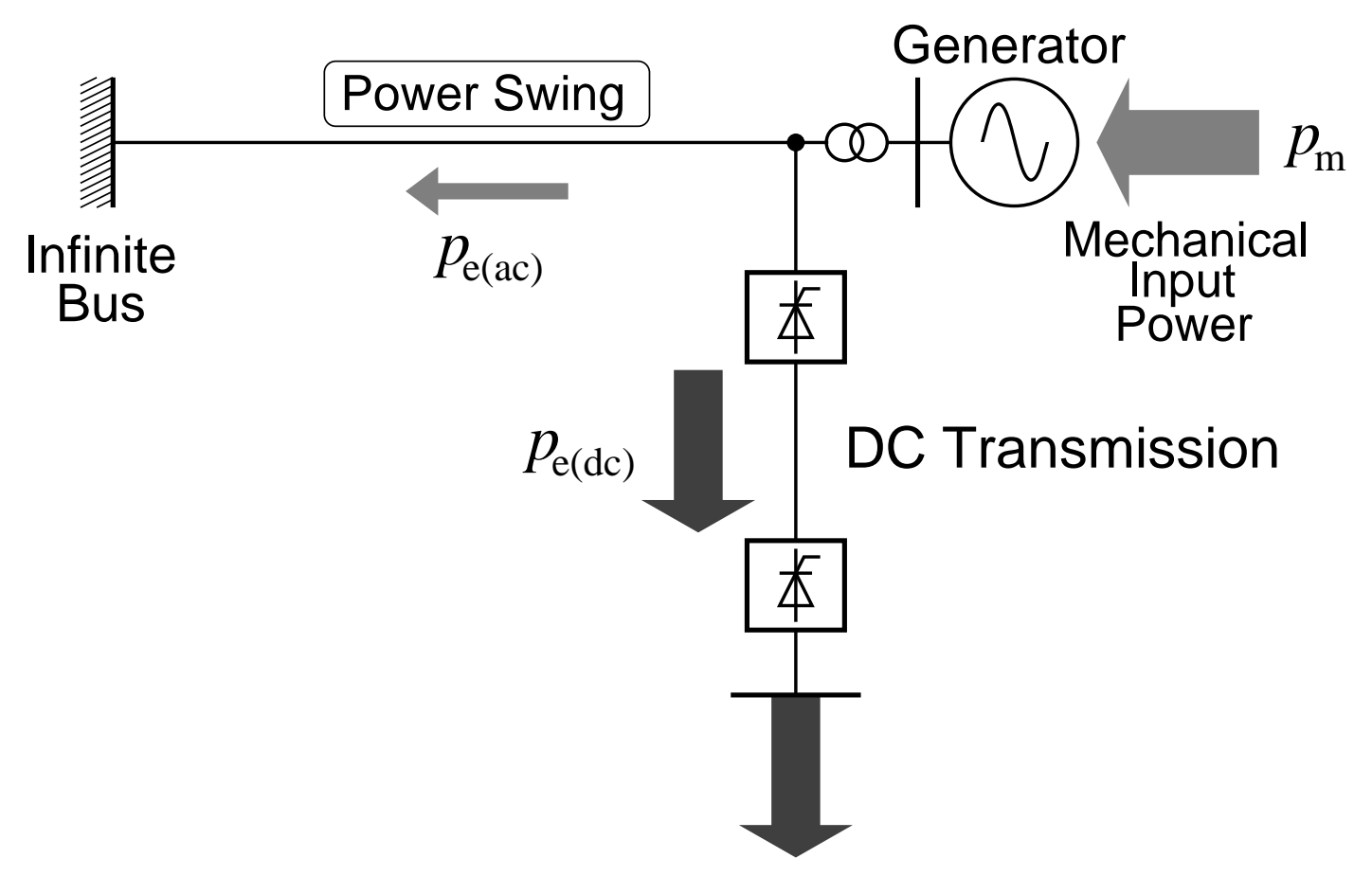

Figure 1: 


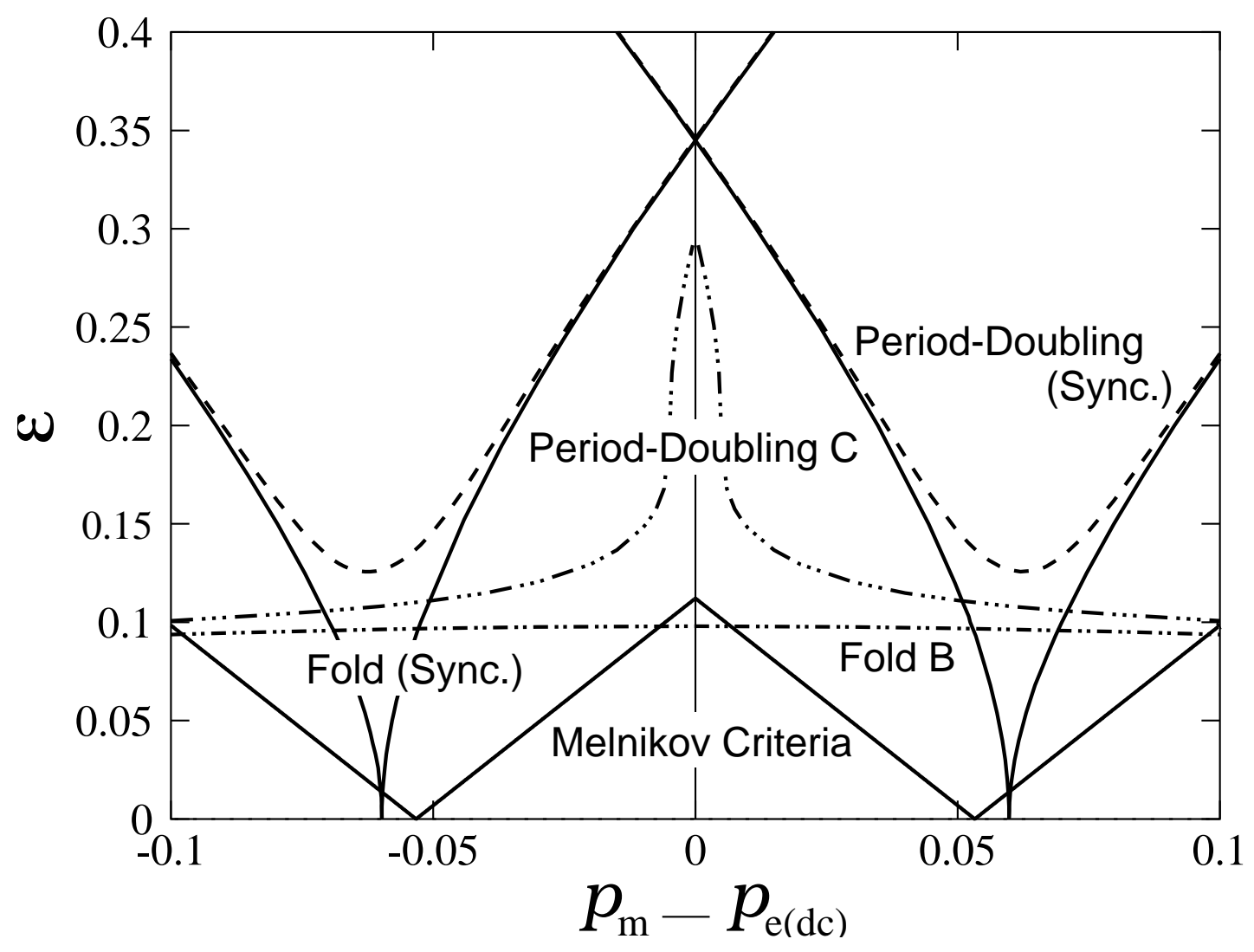

Figure 2: 


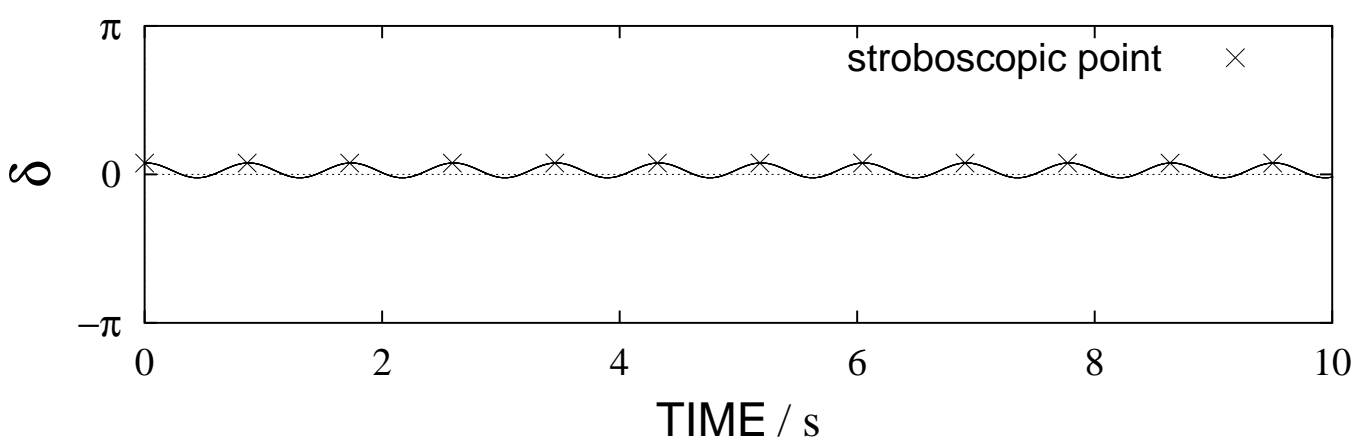

(a)

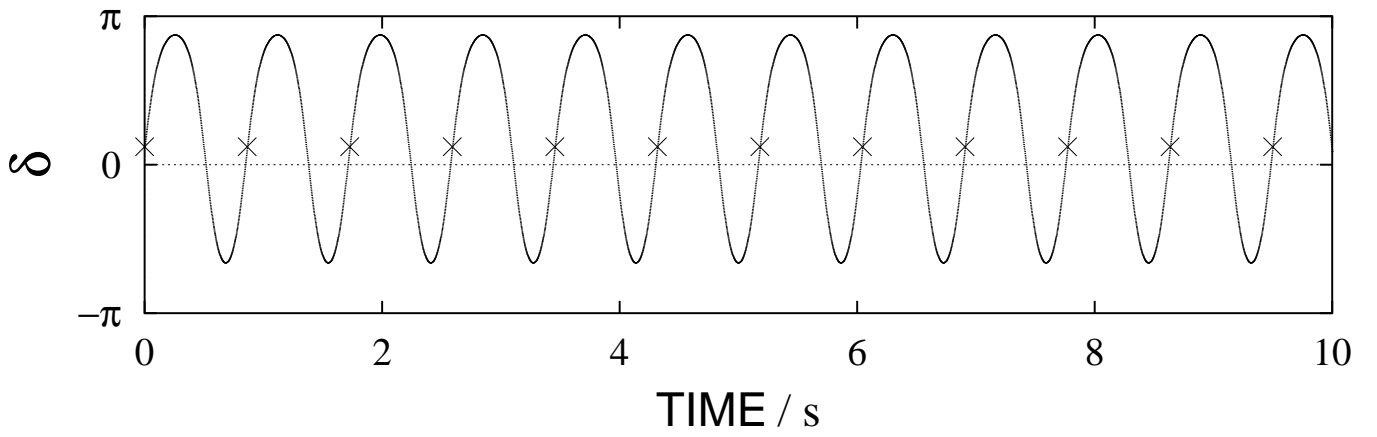

(b)

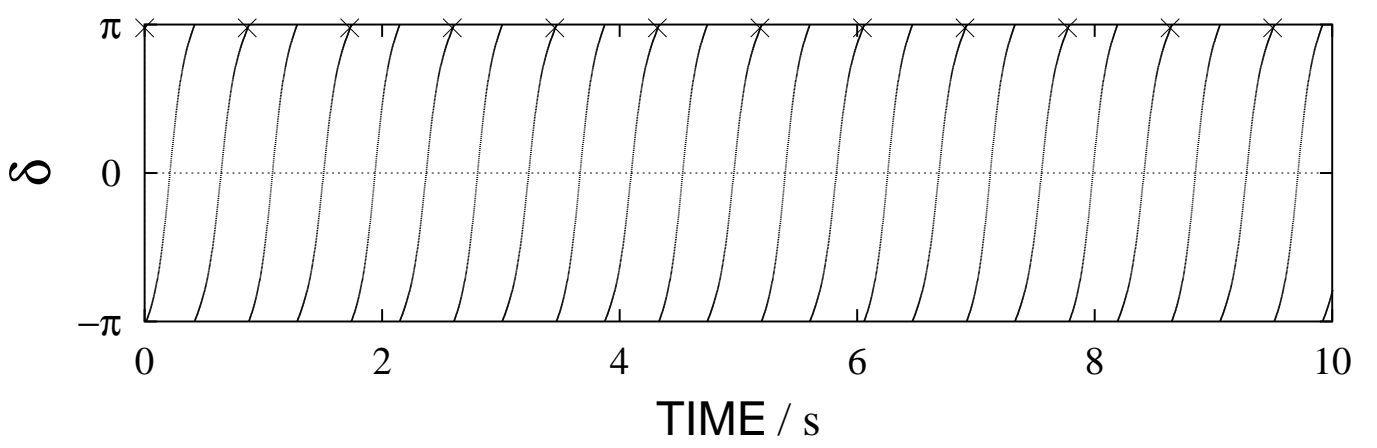

(c)

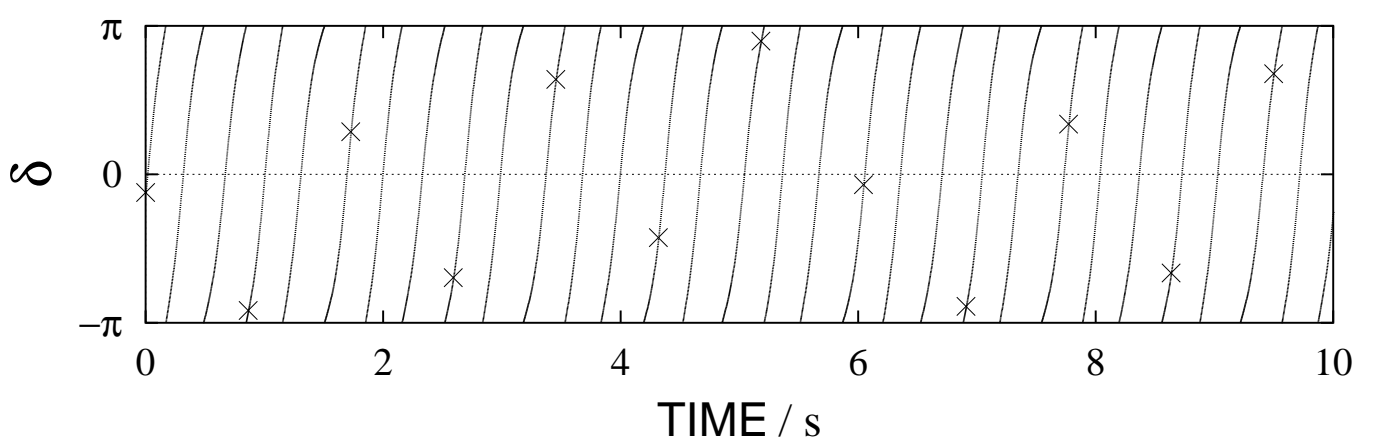

(d)

Figure 3: 

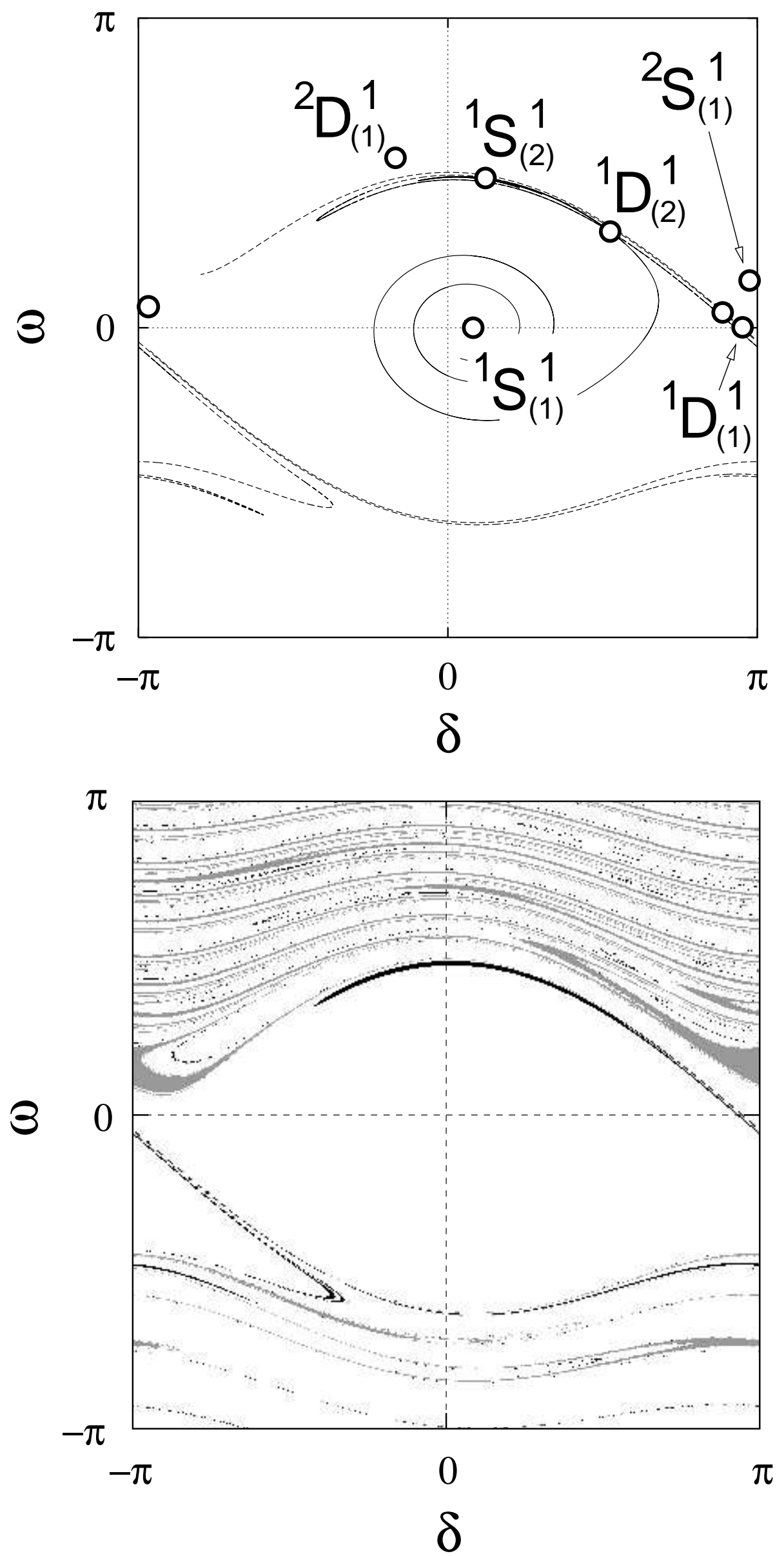

(a)

Figure 4: 

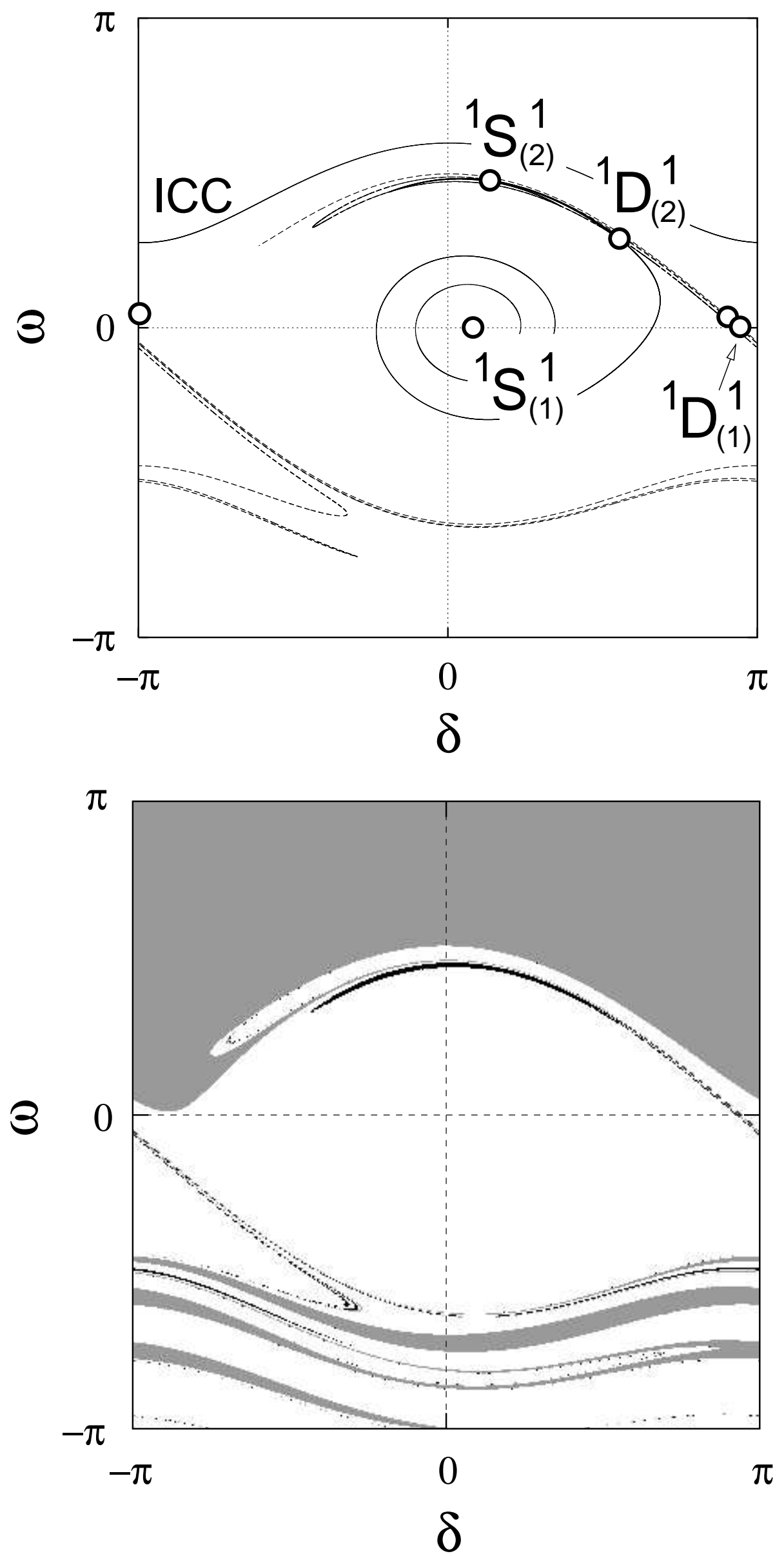

(b)

Figure 4: 


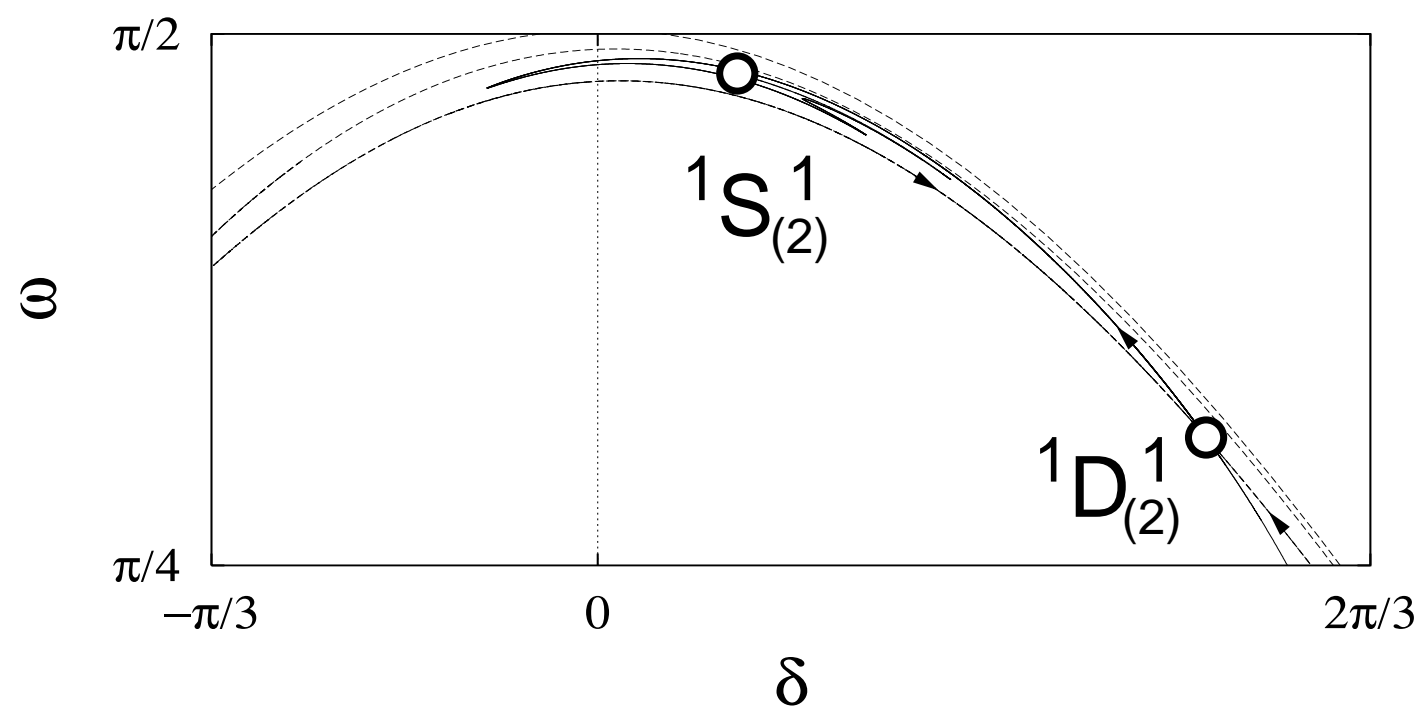

(a)

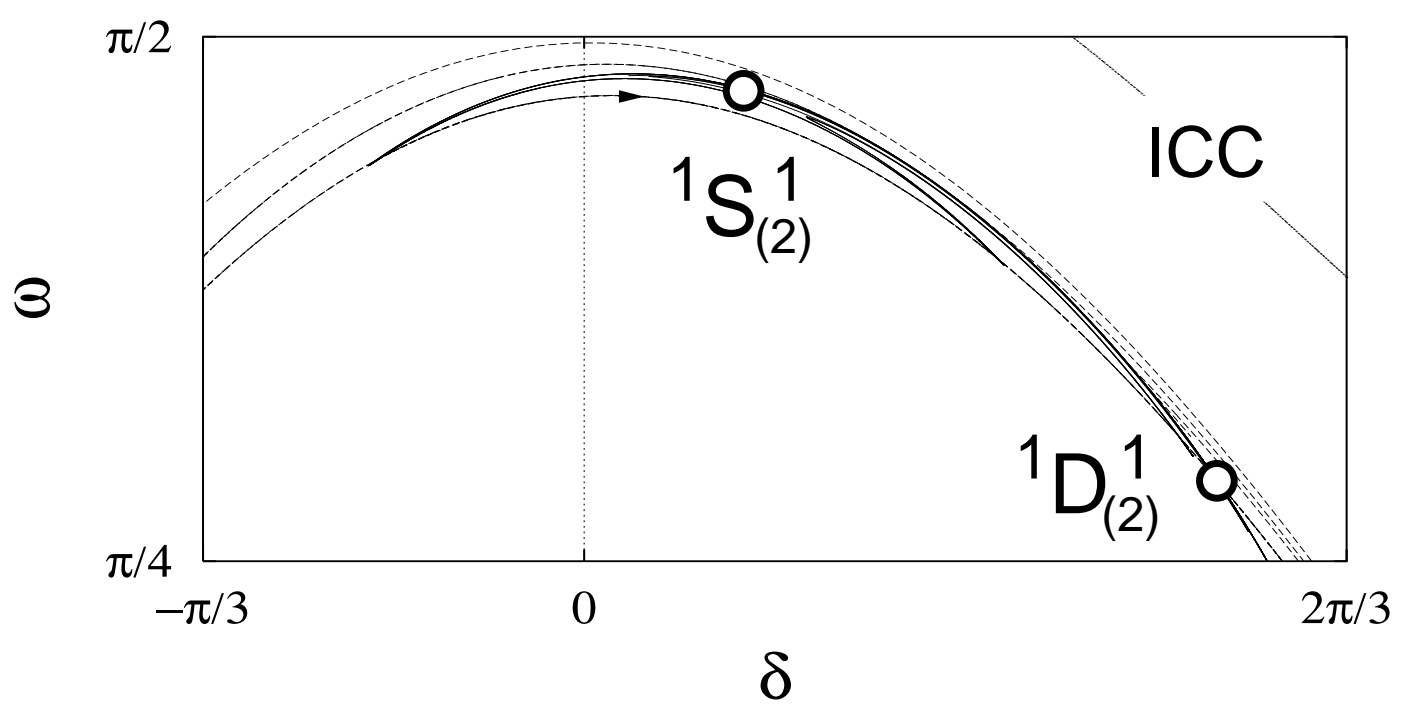

(b)

Figure 5: 

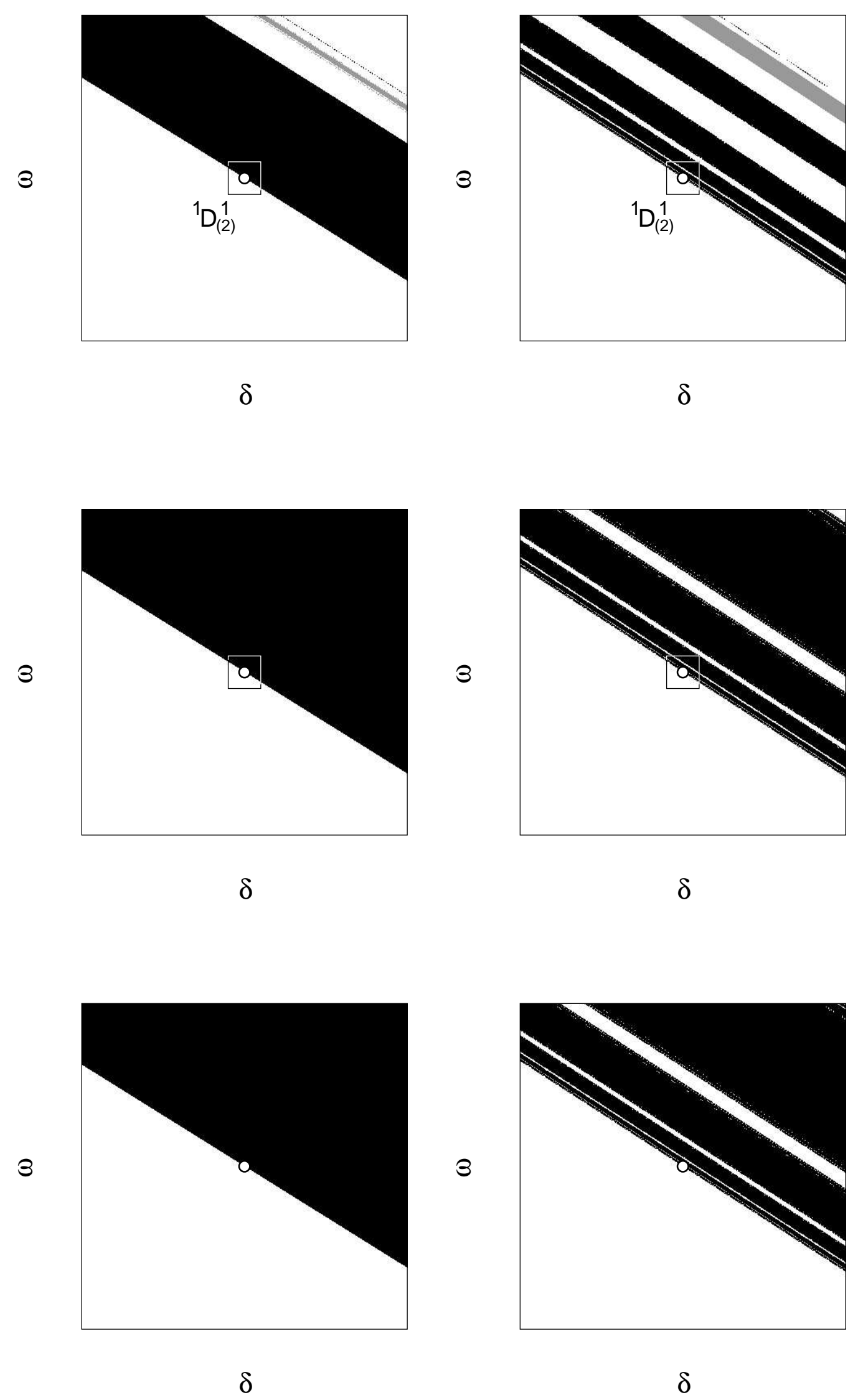

(a)

(b)

Figure 6: 